\title{
Diseño tecnopedagógico de un e-portafolio en educación superior para la mejora de las competencias transversales y la inserción socioprofesional
}

\section{Vanessa Soria ${ }^{a}$}

aUniversidad Pompeu Fabra, Centro para la Innovación en Aprendizaje y Conocimiento (CLIK), Barcelona, vanessa.soria@upf.edu

\begin{abstract}
This article shows how UPF implemented the use of e-portfolios in some of its degrees. The e-portfolio is a document designed with the Mahara tool that compiles all the educational and job-related competences that students acquire throughout their studies. At UPF, students elaborate their e-portfolios in tutoring sessions. The competences included in them help teachers identify what specific aspects must be tackled during their courses. The goal of the eportfolio is to help students acquire the competences they lack while they create a useful resource exportable in and outside the University.
\end{abstract}

Keywords: transversal skills, employability, e-portfolio, portfolio, Mahara, higher education

\section{Resumen}

El presente artículo muestra como se ha diseñado un e-portafolio a través de la plataforma Mahara, aunque el modelo es compatible con otros dispositivos. Este portafolio pretende recoger, mostrar y promover la adquisición de competencias transversales así como de competencias para la inserción socioprofesional durante el tiempo que el alumnado realizada el grado. Así mismo, este e-portafolio se desarrolla paralelamente al programa de tutorías de la UPF, permitiendo la integración de dichas temáticas en la formación específica de los futuros profesionales. El objetivo es mejorar la adquisición de las competencias transversales a la vez que facilitar la creación de un producto final que se pueda mostrar dentro y fuera de la universidad.

Palabras clave: competencias transversales, inserción laboral, e-portafolio, portfolio, Mahara, educación superior 
Diseño tecnopedagógico de un e-portafolio en educación superior para la mejora de las competencias transversales y la inserción socioprofesional.

\section{Introducción}

Uno de los supuestos de la nueva situación del Espacio Europeo de Educación Superior (EEES) es el fomento de las competencias transversales de los estudiantes. Así, los alumnos además de las competencias específicas de sus respectivos grados deberían lograr las competencias transversales definidas para su profesión y formación.

\section{Competencias transversales y evaluación formativa}

La definición de competencia transversales como comentan Aguado, González, Antúnez y Dios, 2017: 130 es: "se entienden las competencias como repositorios de conducta diferenciales que llevan a las personas a desarrollar con mayor eficiencia su trabajo (Sparrow, 1997) y que se componen del conjunto de comportamientos que son instrumentales para la consecución de un desempeño exitoso en el puesto (Bartram, 2005)". Estos mismos autores señalan que dichas competencias son aquellas generales que se pueden aplicar a cualquier puesto de trabajo, ello lo derivan del proyecto Turning Education Structures in Europe de González y Wagenaar (2003). En el marco español, así como las competencias básicas están definidas por el Ministerio de Educación en el real decreto 1393/2007, de 29 de octubre, por el que se establece la ordenación de las enseñanzas universitarias oficiales, en el caso de las competencias generales y transversales suele ser más flexible y se define por la universidad desde cada grado teniendo en cuenta el perfil profesional. Éstas suelen ir relacionadas con aspectos instrumentales (cognitivos, metodológicos, tecnológicos y lingüísticos), interpersonales (interacción social y cooperación) y sistemáticos (uso de las competencias instrumentales e interpersonales) según Aguado, González, Antúnez y Dios (2017: 131). Este enfoque basado en competencias ha permitido mejorar los procesos de enseñanza-aprendizaje y evaluación en la universidad (Vivel-Búa et al. 2015 en Aguado, González, Antúnez y Dios, 2017). Aún asi, como indican los mismos autores, para una implementación exitosa de las competencias son necesarios dos aspectos fundamentales "El desarrollo de un marco común de competencias transversales para los universitarios, y de otro, el desarrollo de instrumentos de medición de dichas competencias" (Aguado, González, Antúnez y Dios, 2017:132). En esa línea de medición o evaluación, hablamos de diferentes tipos, es decir, evaluación diagnóstica o inicial, formativa y sumativa. En el marco del plan Bolonia, se ha equilibrado la evaluación sumativa con la formativa, de este modo, ya no solo se evalúa al final del proceso, sino también durante el mismo para un mayor logro de las competencias, los objetivos y los resultados de aprendizaje. Por tanto, podríamos concluir señalando que la incorporación, específicamente, de la evaluación formativa ha sido y es un valor añadido en la formación, ya que diseña procesos de enseñanza-aprendizaje constantes y activos desde un enfoque constructivista (García-Carpintero, Siles,

(cc) EY-NC-ND 2018, Universitat Politècnica de València

Congreso IN-RED (2018) 
Martínez, Martínez, Manso y Álvarez, 2017) lo que mejora notablemente dichos procesos. Como indican los mismos autores y autoras, esto se da con el cambio de un modelo clásico centrado en el profesor a otro que emplea nuevas metodologías y donde el estudiante es autónomo y responsable de su aprendizaje. Para ello, se debe fomentar la capacidad de autoaprendizaje con el acompañamiento de expertos/as (García-Carpintero, Siles, Martínez, Martínez, Manso y Álvarez, 2017) y en ese contexto deben variarse las metodologías y los sistemas de evaluación que van más allá de los exámenes finales.

\section{El portafolio electrónico}

Dentro de los sistemas de evaluación, encontramos el portafolio: "una colección útil del trabajo de los estudiantes que ilustra los esfuerzos, progresos y logros en una o más áreas" (Barret, 2006 en Soria y Carrió, 2016:385). Cuando hablamos de portafolios electrónicos, esto se refiere al uso de la tecnología como plataforma para desarrollarlos (Soria y Carrió, 2016). El portafolio es un instrumento que se basa tanto en el producto final como en el proceso, por ello se caracterizan por ser herramientas de evaluación formativa y sumativa, o final, de las más empleadas en educación superior en la actualidad. El portafolio como indica López-Pastor (2009) es un instrumento que fomenta una evaluación orientada al aprendizaje y lo que se busca es que:

Los estudiantes sean conscientes de los cambios producidos a lo largo de su proceso de aprendizaje, que autorregulen su forma de aprender y reflexionen sobre los beneficios y dificultades encontradas para poder así ayudarles y estimularles. Esto supone un proceso de aprendizaje que toma en cuenta los conocimientos previos de los estudiantes, su involucramiento en la construcción de su propio aprendizaje y su implicación motivacional, adquiriendo así un aprendizaje más significativo (García-Carpintero, Siles, Martínez, Martínez, Manso y Álvarez, 2017:26).

En esta idea, se le da mucha importancia a la evaluación formativa por: 1) revisión de las actividades del portafolio antes de la entrega final, 2) detección y corrección de las deficiencias del proceso de enseñanza-aprendizaje a lo largo del curso, y 3) evaluación final de la experiencia para mejorar los procesos de enseñanzaaprendizaje para el siguiente curso académico (Pérez Gutiérrez, M. 2017:84). En conclusión, como indican García-Carpintero, Siles, Martínez, Martínez, Manso y Álvarez, (2017:27) el portafolio es una herramienta educativa potente ya que incide en la enseñanza, en el aprendizaje y en la evaluación:

- Enseñanza: retroactividad, centrado en el aprendizaje, interés en el proceso.

- Aprendizaje: crítico-reflexivo, autónomo, responsable, significativo. 
- Evaluación: formativa y sumativa, multidimensional, individualizada y autoevaluativa.

En resumen, como señala (Veytia, 2017:123) el uso de portafolio electrónico integra el diseño tecnopedagógico, la evaluación reflexiva y el desarrollo de competencias, lo que hace de él una herramienta muy completa y pertinente en la Educación Superior actual.

Cabe destacar, que el e-portafolio, como evidencia formativa y evaluativa, muestra las competencias adquiridas pero también puede ayudar especialmente en el proceso de inserción socioprofesional, demostrando a las entidades externas el perfil del estudiante. Es decir, aquello que saeb hacer de una forma visual y práctica.

\section{La inserción socioprofesional}

Así mismo y para concluir, destacar que la formación de los estudiantes universitarios suele ir encaminada a facilitarles, una vez finalizados sus estudios, la inserción sociprofesional, aspecto que como destacan Ruiz y Molero, 2002 (en Jiménez, 2009:1) es cada vez más transcendental para "jóvenes, empresas, universidad y sociedad" ya que:

La flexibilización del mercado de trabajo, los avances tecnológicos, económicos y demográficos junto a la democratización de la universidad y el aumento de otras posibilidades formativas, ha originado que la preocupación por la búsqueda de un empleo adecuado a una cualificación determinada sea una constante en las personas que obtienen una titulación. (Jiménez, 2009:1)

Por tanto, una de las competencias a trabajar es el acceso al mundo profesional. Éste es un aspecto que aunque fundamental, no se trabaja en asignaturas concretas pero si de forma transversal durante el grado en algunos caso. Aun así, es necesario que la universidad ofrezca herramientas y estrategias para encarar el futuro profesional más allá de las competencias específicas sino también teniendo en cuenta las competencias generales y transversales. Es decir, "el nuevo modelo de educación superior destaca por la importancia concedida a las competencias profesionales y la empleabilidad como elemento de cambio.". Siguiendo con el mismo tema citan "los estudiantes esperan que las universidades los apoyen, no solo en su desarrollo intelectual y académico, sino también estableciendo una plataforma para el empleo y el desarrollo de la carrera". (García-Álvarez, Vázquez-Rodríguez, Mella-Núñez, 2017:45). 


\section{Objetivos}

El objetivo de esta experiencia es mejorar la adquisición de dichas competencias (transversales y socioprofesionales) a la vez que facilitar la creación de un producto final que se pueda mostrar dentro y fuera de la universidad.

\section{Desarrollo de la innovación}

Durante el curso 2017-2018 se ha implementado un e-portafolio en el Grado en Global Studies de la Universidad Pompeu Fabra. Para ello durante el tercer trimestre del cuso 2016-2017 se realizó un diseño previo, posteriormente validado por los coordinadores del grado. A continuación se expone el diseño pedagógico y algunes detalles de la implementación.

\section{- El diseño pedagógico}

\section{Contextualización:}

La Universidad Pompeu Fabra cuenta con un centro de apoyo al profesorado en temas pedagógicos y de innovación docente. Este es denominado Centro para la Innovación en Aprendizaje y Conocimiento (CLIK) (www.upf.edu/web/clik). El CLIK ofrece recursos de apoyo al profesorado en diferentes áreas: formación, multilingüismo, nuevas metodologías, innovación tecnológica,entre otros temas. Así, es habitual que el profesorado de los diferentes estudios, solicite asesoramiento para la implementación de nuevas propuestas que mejoren los procesos de enseñanza-aprendizaje. Concretamente, en este caso hablamos de la incorporación de un portafolio electrónico para la mejora y visibilización de las competencias transversales de un grado específico.

Como se ha comentado con anterioridad, señalar que un portafolio es una recopilación de información y documentación representativa de un proceso de aprendizaje. Así, el e-portafolio (portafolio electrónico) se diferencia por tener las mismas carácterísticas pero además es digital y utiliza plataformas en línea. En el caso de la UPF, desde el curso 2016-2017, se hace uso del portafolio electrónico Mahara, ya que está integrado en la plataforma virtual de la universidad (denominada Aula Global).

El grado específico, objeto de la implementación, es el Global Studies (Grado en Estudios Globales), que se imparte en su mayoritariamente en inglés. Éste tiene una duración de 3 años, se organiza desde la Facultad de Humanidades y su objetivo es "Estos estudios están pensados como una oferta docente abierta a estudiantes de todo el mundo interesados en entender aquellos fenómenos — culturales, económicos, jurídicos, medioambientales, políticos, sociales y tecnológicos- que nos afectan 
Diseño tecnopedagógico de un e-portafolio en educación superior para la mejora de las competencias transversales y la inserción socioprofesional.

con independencia de nuestra precisa ubicación territorial y cuya comprensión precisa de una aproximación transdisciplinar y una visión global del ser humano."

A continuación destacamos algunos de los recursos audiovisuales de difusión sobre dicho grado:

- Información general: https://www.upf.edu/web/graus/grau-en-estudisglobals

- Video de presentación: https://www.youtube.com/watch?time continue $=12 \& v=t$ cuAcylhPp0

- Video informativo: https://www.youtube.com/watch?v=GJQsn3TLwyM

Cabe destacar que, aunque la propuesta de diseñar el e-portafolio tanto a nivel de contenido como de forma, se inició en dichos estudios, la propuesta es extrapolable a cualquier grado ya que hablamos de competencias que deben adquirir todos los estudiantes universitarios y que no dependen de la titulación ya que son transversales.

En esa línea, en la UPF ya partíamos de un portafolio de éxito implementado durante años en el grado de Biología Humana de la Facultad de Ciencias Experimentales y de la Salud (CEXS) (https://www.upf.edu/web/biomed/grau-en-biologia-humana). Éste se caracterizaba por desarrollarse durante los 4 años del grado. En el primer curso se presentaba y se empezaba a trabajar en él y a partir del segundo a través de las tutorías trimestrales. Apuntar que el programa de tutorías de la UPF (ACTE) también es una seña de calidad y éxito. El ACTE se basa en que cada estudiante, al iniciar sus estudios, tiene asignado un tutor/a que es profesor/a de su grado. Con este tutor se reúnen una vez al trimestre para comentar como van sus estudios y si necesitan algún tipo de apoyo. Por ello, trabajar el portafolio en este contexto es ideal y se mantiene este diseño/sistema para el portafolio del Global Studies. La presentación del portafolio se realiza a través de una sesión específica informativa para tutores y estudiantes por separado, asimismo se les envía una guía muy práctica con toda la información. Dicha guía ofrece una serie de recursos, además de informaciones clave, como unas fichas para ir evaluando las competencias tutoría a tutoría y una muestra del producto final que se pide. El trabajo en las tutorías se realiza a través de revisar las competencias transversales estipuladas, en este grado: trabajo en grupo, comunicación oral, comunicación escrita y búsqueda de información. Para ello, en una de las tutorías del año se revisa como van estas competencias haciendo uso de la ficha anteriormente citada. Éstas fichas se basan en analizar qué potencialidades y dificultades tienen en cada competencia, cómo pueden mejorar y en qué acciones concretas van a hacerlo. Las fichas son firmadas por el tutor/a y el tutorando/a y son evidencias para el portafolio que se presenta al final. El producto final es una página de reflexión por competencia, haciendo asociaciones

(c)) EY-NC-ND 2018, Universitat Politècnica de València

Congreso IN-RED (2018) 
entre la evolución de los diferentes cursos y las fichas que lo demuestran, así como con muestras de tres trabajos por competencia que denoten la evolución. Finalmente, si el portafolio es apto tienen la suma 0.5 de la nota de una asignatura del último año del grado.

Este modelo de éxito nos permitió mantener algunos aspectos para la nueva propuesta:

- Desarrollo durante todos los cursos del grado

- Enlazar el portafolio con el programa de tutorías

- Trabajar las competencias transversales indicadas, aunque se le añadieron algunas más específicas del grado en Global Studies

- Mantener la guía, las fichas y el producto final, con algunas mejoras y traducida al inglés

- Sumar un pequeño porcentaje en la nota de una asignatura del último año de grado.

Aspectos a mejorar:

- Modificar el formato del portafolio: pasarlo de papel a digital para permitir nuevos productos a añadir, por ejemplo videos, también para recoger los feedbacks de los tutores durante todo el desarrollo y para mostrarlo en entrevistas de trabajo.

- Añadir competencias transversales relacionadas con el grado específico como se ha indicado: intercultura y acceso a la profesión

- Incorporar aspectos de inserción laboral

- Mejorar las fichas de seguimiento, partiendo de las existentes, pero añadiéndole aspectos del coaching educativo, rúbricas y otros elementos para un análisis más profundo.

- Facilitar los comentarios del tutor en cada uno de los productos del portafolio para mejorar el feedback y el aprendizaje

- Colaborar con el serviciode carreras profesionales de la universidad, para entender el portafolio como un proyecto integral del grado y de la universidad.

\section{El portafolio y su contenido}

En resumen, el e-portafolio del grado en Global Studies está orientado en dos líneas: un bloque demostrativo del logro de las competencias transversales destinado al curriculum laboral y otro bloque para la inserción socioprofesional.

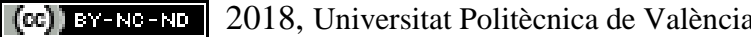


Concretamente, por lo que respecta a las competencias transversales finalmente a trabajar en el e-portafolio de Global Studies, y siguiendo la clasificación del programa Turning, son:

- Competencias transversales instrumentales: comunicación oral y comunicación escrita.

- Competencias transversales interpersonales: trabajo en grupo e intercultural

- Competencias transversales sistemáticas: búsqueda de información y acceso a la ocupación.

Estos bloques se traducen en el siguiente contenido demostrativo del perfil del estudiante:

a) Video curricular de presentación del estudiante (bloque inserción socioprofesional)

b) Carta de presentación del estudiante (bloque inserción socioprofesional)

c) Carta de recomendación del tutor u otras que pueda tener durante el grado(bloque inserción socioprofesional)

d) Curricular en formato infografía (bloque inserción socioprofesional)

e) Carpetas de cada competencia transversal con las fichas, la hoja resumen de la evolución durante el grado y productos como evidencias de la adquisición de las competencias. También se le añadió un extra "otras" por si algún alumno quería añadir más evidencias. (bloque competencias transversales)

f) Además, se le añadió un apartado con datos de contacto.

\section{El portafolio electrónico y el uso de Mahara}

Como se ha indicado, el contenido del portafolio recibió pequeñas mejoras en cuanto a las competencias, siguiendo el modelo del Grado en Biología Humana pero añade una parte de inserción profesional hasta ahora inédito en la universidad. Aunque la mayor disrupción tiene que ver con su formato, el paso de papel a digital. Básicamente, esto ofrece:

- Recibir feedback a través de comentarios que pueden ser públicos o privados en cada una de las piezas del portafolio.

- Consultar el portafolio en cualquier momento por parte del alumnado, profesorado, compañeros o personas externas.

- Incorporar nuevos productos más visuales y atractivos. Por ejemplo, videos o infografías.

- Facilitar la muestra desde cualquier dispositivo a agentes internos y externos. 
- Utilizar las piezas o productos del portafolio de manera separada o subiéndolos en otras aplicaciones: LinkeIn, blogs, etc.

- Tener en cuenta aspectos de protección de datos, en una versión de Mahara cerrada para la UPF e incorporada e integrada en la plataforma virtual.

- Ser más sostenibles reduciendo el uso de papel.

A continuación, mostramos el portafolio que hicimos como plantilla para el alumnado. Pueden acceder todos los estudiantes a Mahara y adquirir la plantilla o empezar a elaborar una propia (ver imagen 1).

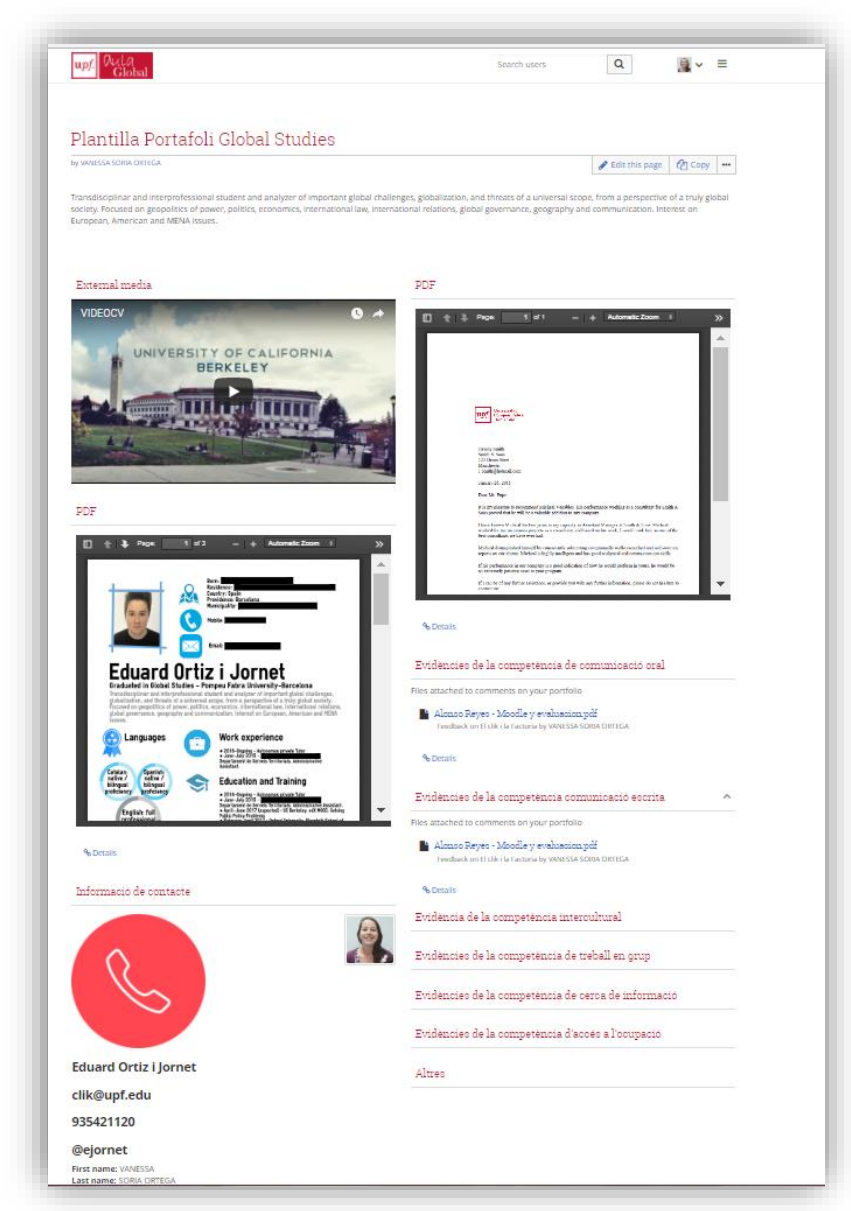

Imagen 1. Plantilla del e-portafolio del Grado de Global Studies de la UPF

\section{Las fichas para realizar el seguimiento de las competencias}

Por lo que respecta a la ficha, partíamos de un documento donde habían los títulos de los temas a trabajar "puntos fuertes, puntos débiles, propuesta de mejora y 
Diseño tecnopedagógico de un e-portafolio en educación superior para la mejora de las competencias transversales y la inserción socioprofesional.

actividad donde aplicar la mejora". Se decidió diseñar un modelo más atractivo, incorporando preguntas basadas en el coaching educativo, así como una rúbrica para analizar la adquisición de la competencia de una manera más detalla, sencilla y profunda. Así mismo, se preparó una ficha didáctica para el profesorado con el objetivo de informar y dar recomendaciones para usarla de la forma más eficaz posible y también decidimos ofrecer la ficha en versión PDF de autorelleno para colgarla en el e-portfafolio. A continuación se muestra un ejemplo de ficha de competencias.

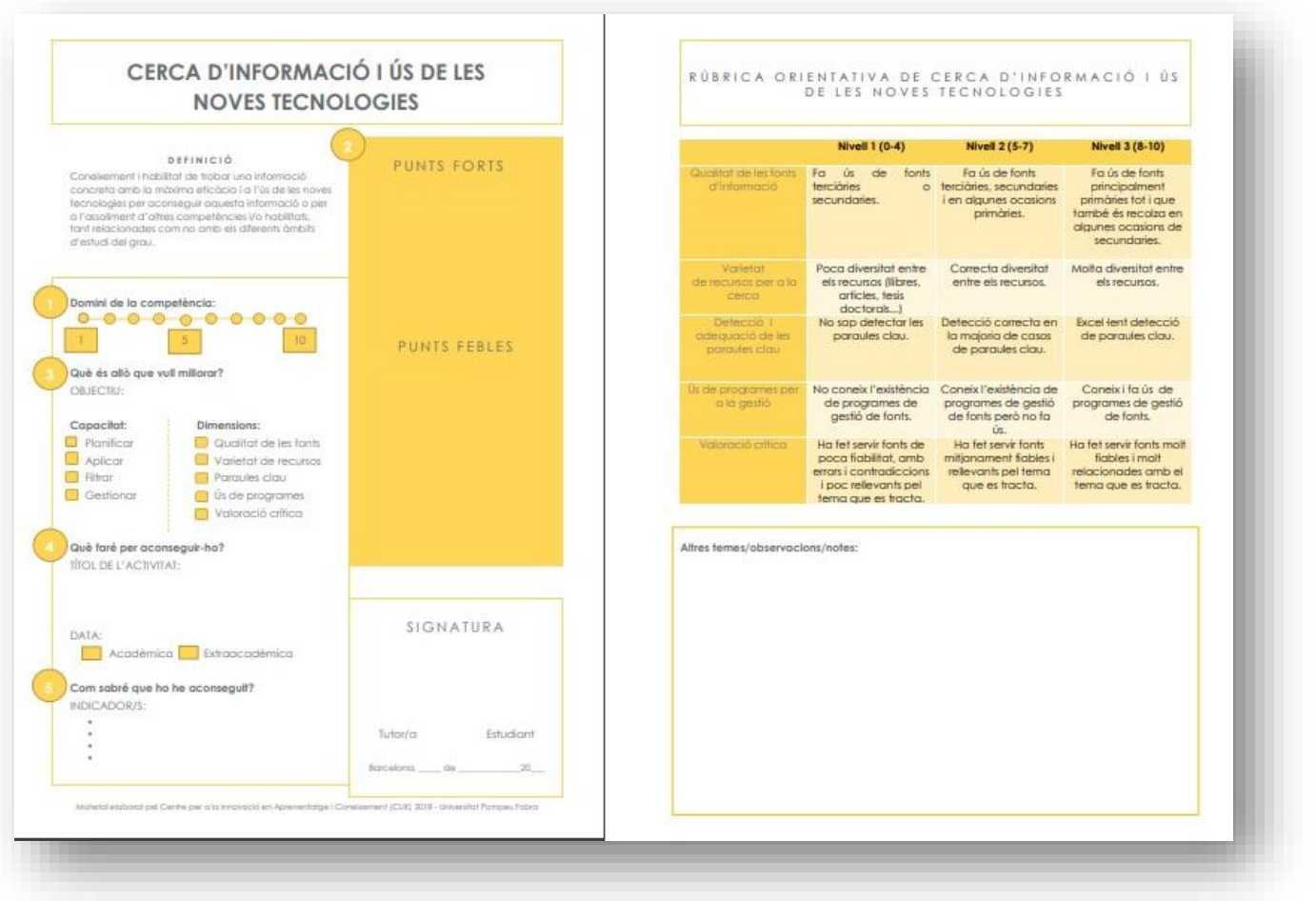

Imagen 2. Ficha de competencia oral.

\section{El proceso de validación e implementación:}

Para aplicación del portafolio, seguimos los pasos detallados a continuación:

1. Diseño junto a un estudiante del grado y la coordinación de los estudios

(cc) EY-NC-ND 2018, Universitat Politècnica de València

Congreso IN-RED (2018) 
2. Propuesta de diseño y validación, tanto de contenido como de formato

3. Elaboración de la plantilla modelo y guía para estudiantes

4. Sesiones informativas para alumnado y profesorado

5. Inicio de la elaboración del portafolio en las tutorías

6. Curso 2018-2019 evaluación del primer curso como prueba piloto.

\section{Resultados}

Por ahora podemos valorar la acogida de profesorado y alumnado, la cual ha sido muy positiva. El diseño además ha permitido enlazar cursos relacionados con el acceso a la ocupación que están desarrollando desde el servicio de carreras profesionales de la universidad. Por lo que respecta a las limitaciones podemos indicar que destacamos las siguientes:

- Limitaciones a la hora de mantener el portafolio una vez se gradúen los estudiantes, ya que va asociado al correo de alumno. Estamos trabajando para buscar modos de solucionar este aspecto. Por ahora hemos logrado que lo tengan un año más después de graduarse.

- La capacidad de espacio de Mahara es muy limitada, así requiere de usar otros lugares de hospedaje: YouTube. Drive, Dropbox, entre otros. Por tanto, ofrecemos una guía de como usar Mahara complementado con otras aplicaciones para aprovechar los megas reducidos que ofrece Mahara. También se contempla pedir una ampliación de espacio.

- El diseño del portafolio en cuanto a estética es común para todos los estudiantes y no permite customización.

\section{Conclusiones}

En cuanto al diseño, podemos valorar que éste da respuesta a las necesidades educativas actuales, a las competencias transversales, así como a la formación en materia de inserción profesional, algo que ayuda enormemente a la incorporación en el mercado laboral de nuestros futuros ingresados.

Aun así, y aunque partíamos de un modelo de éxito ya testado, faltarán varias generaciones de estudiantes del grado en Global Studies para realizar mejoras específicas y adaptar la propuesta de portafolio electrónico a sus necesidades educativas, formativas y laborales. La versión digital es una mejora, pero seguro que en los próximos años se desarrollan formatos más diruptivos de portafolios electrónicos que respondan a las necesidades educativas y profesionales. 
Diseño tecnopedagógico de un e-portafolio en educación superior para la mejora de las competencias transversales y la inserción socioprofesional.

\section{Referencias}

Aguado, D.; González, A.; Antúnez,M. y Dios, T. (2017). Evaluación de Competencias Transversales en Universitarios. Propiedades Psicométricas Iniciales del Cuestionario de Competencias Transversales. REICE. Revista Iberoamericana sobre Calidad, Eficacia y Cambio en Educación, 2017, 15(2), 129-152.

García-Álvarez, J.; Vázquez-Rodríguez, A.; y Mella-Núñez, I. (2017). La empleabilidad de los universitarios en Europa. Contribuciones de la educación no formal. Revista de estudios e investigación en psicología y educación. Vol. Extr. $N^{\circ} 7$. ISSN: 2386-7418.

García-Carpintero, E.; Siles, J.; Martínez, M.E.; Martínez, E.; Manso, C. y Álvarez, B. (2017). Metodologías de enseñanza-aprendizaje en enfermería: ¿es el portafolio una metodología acorde con el Espacio Europeo de Educación Superior?. Revista Enfermería Docente; enero-junio (108): 24-28 ISSN 2386-8678.

Jímenez, A. (2009). Reflexiones sobre la necesidad de acercamiento entre la universidad y mercado laboral. Revista Iberoamericana de Educación. N50/1- 25 de julio. ISSN: 1681-5653.

López-Pastor, V.M. (coord.). (2009). Evaluación formativa y compartida en Educación Superior. Propuestas, técnica, instrumentos y experiencias. Madrid: Narcea

Pérez Gutierréz, M. (2017). Diseño de una experiencia de evaluación formativa mediante portafolio en el grado en magisterio de educación infantil en la Universidad de Cantabria. Infancia, educación y Aprendizaje (IEYA). Vol.2, N $N^{\circ}$ (edición especial), pp.82-87- ISSN:0719-6202

Soria, V. y Carrió, M. (2016). Pedagogías disruptivas para la formación inicial de profesorado: usando blogs como e-portafolio. Revista de currículum y formación del profesorado. Vol. 20, nº2 (mayo-agosto). ISSN 1138-414X

Veytia, M.G. (coord..). (2017). Diferentes miradas sobre el empleo de las tecnologías de la información y la comunicación en educación. Durango: México 